\title{
STM study of fractal scaling in evaporated gold films
}

\author{
L. Vázquez ${ }^{a}$, R.C. Salvarezza ${ }^{b}$, P. Herrasti ${ }^{c}$, P. Ocón ${ }^{c}$, J.M. Vara ${ }^{c}$ and A.J. Arvia ${ }^{b}$ \\ a Instituto de Ciencia de Materiales, CSIC, Departamento de Física Aplicada, C-XII, Universidad Autónoma de Madrid, \\ 28049 Madrid, Spain \\ ${ }^{b}$ Instituto de Investigaciones Fisicoquímicas Teóricas y Aplicadas (INIFTA), Sucursal 4, Casilla de Correo 16, 1900 La Plata, Argentina \\ c Departamento de Química Física Aplicada C-II, Universidad Autónoma de Madrid, 28049 Madrid, Spain
}

Received 27 July 1992; accepted for publication 18 November 1992

Fractal characterization of vacuum-evaporated gold films on glass substrates with thicknesses $(\bar{h})$ comprised between 30 and 850 $\mathrm{nm}$ is made from STM data by using the dynamic scaling approach for surface growth. The rms roughness $(\xi)$ and the scan length ( $L$ ) obey a $\xi$ versus $I^{\alpha}$ relationship with $\alpha$ depending on $\bar{h}$. For $\bar{h} \geq 500 \mathrm{~nm}$ and $L>d_{\mathrm{s}}$, the average column diameter, $\alpha \simeq 1 / 3$ in agreement with the predictions of ballistic deposition models without restructuring. For $L<d_{\mathrm{s}}, \alpha \simeq 0.9$ approaching a euclidean value. The STM method is verified through its application to several computer-generated surfaces, leading to a good agreement with the theoretical values.

\section{Introduction}

A relatively large number of physical and chemical processes under non-equilibrium conditions are related to the development of complex structures involving rough surfaces. This is the case of metal deposition from the vapour where either atoms or molecules are incorporated to the growing deposit following near-ballistic trajectories [1]. These deposits exhibit a columnar structure [2] with interfaces which have been described either as self-similar [3] or self-affine fractals [4].

Recently attempts to characterize vapour-deposited metal surfaces as fractals from STM imaging analysis [5-10] provided valuable information on the matter. Thus, vapour-deposited gold films were studied by using a perimeter versus area analysis of lakes and islands generated on STM images [9]. This type of analysis allowed $\alpha$ to be obtained, the fundamental parameter for describing the roughness of self-affine surfaces, which is related to the local fractal dimension of the deposit surface, $D_{\mathrm{s}}$, through the following equation [4]:

$D_{\mathrm{s}}=D-\alpha$,

where $D$ is the dimension of the space associated with the growth of the deposit. For a gold film of average thickness $\bar{h}=170 \mathrm{~nm}, \alpha \simeq 0.32$, a figure which is close to $\alpha=1 / 3$, as it is expected for $3 D$ large-scale computer simulations of ballistic models without reconstruction. A similar value of $\alpha$ was obtained for $\bar{h}=850 \mathrm{~nm}$ by using the dynamic-scaling approach for surface growth [5].

In this work we report on STM measurements of vapour-deposited gold films grown under nonequilibrium conditions covering the $30<\bar{h}<850$ $\mathrm{nm}$ range. The dynamic-scaling approach of surface growth [4] applied to STM data resulting from gold films with $\bar{h} \geq 500 \mathrm{~nm}$, allows us to prove the self-affine fractal character of the gold deposit surface and the validity of ballistic models for describing some aspects of vapour-deposited gold film morphology. Otherwise, for $\bar{h}=30 \mathrm{~nm}$, the STM data prove that the film can be considered as a 2D array of smooth gold grains and the fractal dimension of the surface approaches that expected for percolation clusters. 


\section{Experimental}

The preparation of the vapour-deposited gold films has been reported in detail elsewhere $[9,10]$. Briefly, gold films were grown on smooth glass substrates by employing an evaporator chamber under the following conditions: pressure $10^{-4}$ Torr, average deposit growth rate $30 \mathrm{~nm} \mathrm{~s}^{-1}$, and substrate temperature $298 \mathrm{~K}$. The angle between the direction of incident particles and the substrate normal was set in the $2^{\circ}-25^{\circ}$ range. The average film thickness, $\bar{h}$, was measured with a profilometer.

To study the surface morphology of the deposits a piezo-tube STM operating in air was used. The piezo-tube was calibrated by imaging highly oriented pyrolytic graphite (HOPG). The tips were made from $0.5 \mathrm{~mm}$ diameter platinum wires directly by cutting. To minimize occasional tip geometric artifacts different tips were used although no marked influence of the tip shape was detcctcd on the experimental data. STM measurements were made using a $0.05 \mathrm{~V}$ bias voltage with the tip (+) at 1-2 nA constant current. Data were acquired in a fully automated workstation and stored as digitized images with $256 \times 256$ pixels. A more detailed description of STM measurements is given in refs. [9-11].

\section{The method}

The STM method is based on the dynamicscaling approach of surface growth [4,12]. For a sample of unit lateral dimension $L$, the mean square average roughness, $\xi(L)$, is defincd as:

$\xi(L)=\left\{\frac{1}{L} \sum\left[h\left(x_{i}\right)-\bar{h}\right]^{2}\right\}^{1 / 2}$,

where $h\left(x_{i}\right)$ is the deposit height measured along the $x$-direction at the $x_{i}$ position, and $\bar{h}$ is the average height of the sample. Besides, $\xi$ and $L$ are related through the equation:

$\xi(L, h) \propto L^{\alpha} f(x)$,

where $f(x)=\bar{h} / L^{\gamma}$. The function $f(x)$ has the following properties: $f(x)=$ const. for $x \Rightarrow \infty$, and $f(x)=x^{\alpha / \gamma}$ for $x \Rightarrow 0$. After a certain critical thickness (time), $\xi$ reaches a steady-state value, and eq. (3) becomes:

$\xi(L) \propto L^{\alpha}$.

The preceding dynamic-scaling concepts can be applied to STM topographic images as $\xi=$ $\xi_{\text {STM }}$. Accordingly, we have determined the rms value of the fluctuations of $h\left(x_{i}\right)$ over each STM scan segment of length $L=L_{\mathrm{s}}$ along the $x$-direction after plane correction. More explicitly, we have used:

$\xi_{\mathrm{STM}} \propto\left\{\frac{1}{L_{\mathrm{s}}} \sum\left[h\left(x_{i}\right)-\bar{h}_{L_{\mathrm{s}}}\right]^{2}\right\}^{1 / 2}$,

$\xi_{\mathrm{STM}} \propto L_{\mathrm{s}}^{\alpha}$.

The capability of the proposed method for calculating the value of $\alpha$ from STM images has been verified by analyzing several computer-generated self-affine fractal surfaces with preset values of $\alpha\left(\alpha_{\mathrm{th}}\right)$, built on a $256 \times 256$ square grid by using the successive random addition algorithm [13]. The plot of $\alpha_{\mathrm{m}}$ versus $\alpha_{\mathrm{th}}$, where $\alpha_{\mathrm{m}}$ is the value of $\alpha$ obtained by applying the proposed method to these surfaces, is displayed in fig. 1 where the straight line (that expected for the

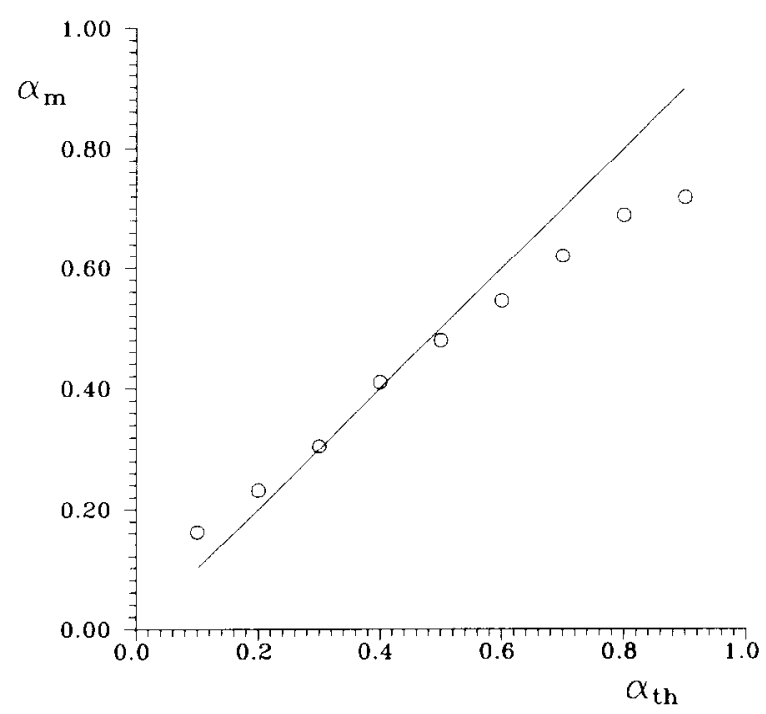

Fig. 1. $\alpha_{m}$ versus $\alpha_{\text {th }}$ plots for surfaces generated by the successive random addition algorithm. Data obtained with the present method $(0)$. The full trace corresponds to the expected line. 
ideal relationship between $\alpha_{\mathrm{m}}$ and $\alpha_{\mathrm{th}}$ ) is used for determining $\alpha_{\mathrm{mc}}$, the corrected value of $\alpha_{\mathrm{m}}$. As already reported [6] a better agreement of data shown in fig. 1 could be obtained by increasing the number of points in the square grid. However, the $256 \times 256$ square grid used for imaging procedure involves a short imaging time, minimizing drift effects.
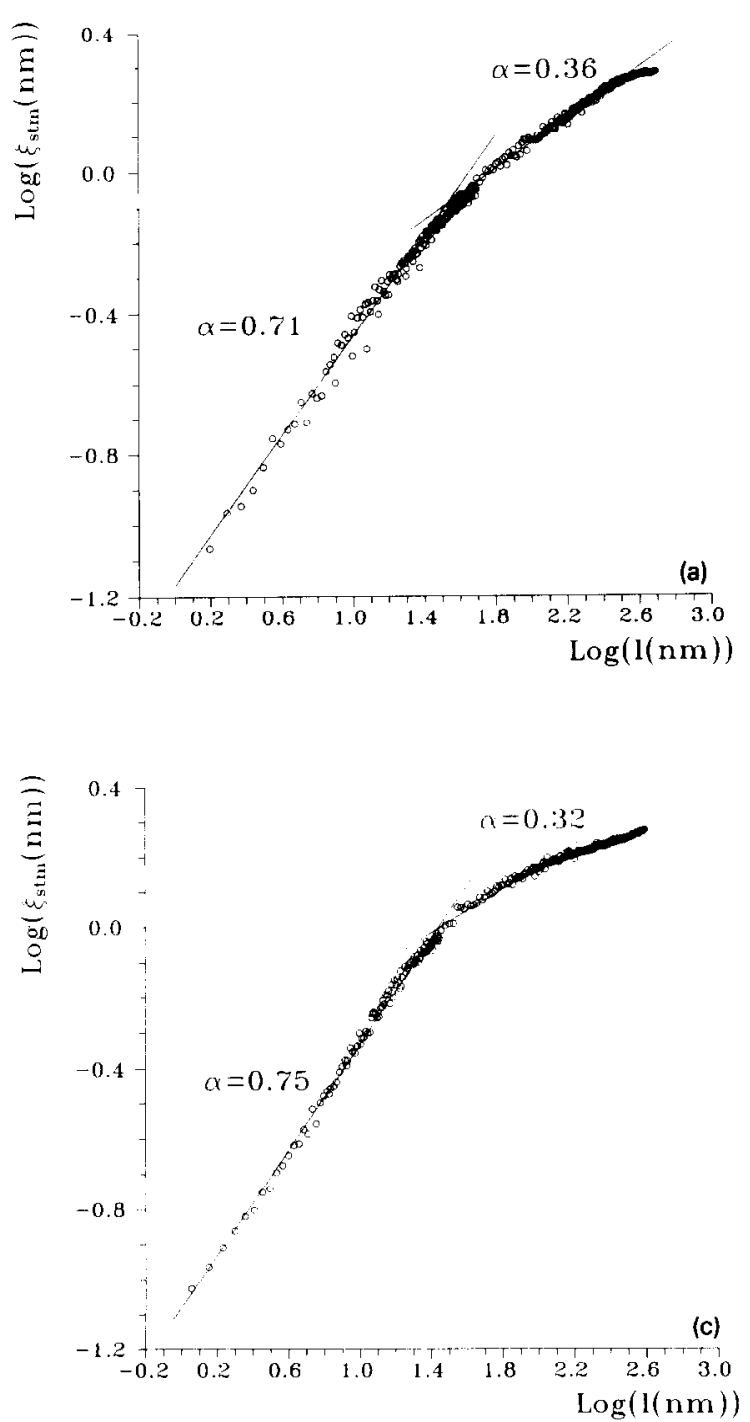

\section{Results and interpretation}

The $\log \xi_{\text {STM }}$ versus $\log L_{\mathrm{s}}$ plots for different vapour-deposited gold surfaces are shown in figs. $2 \mathrm{a}-2 \mathrm{~d}$. For each value of $L_{\mathrm{s}}$ the corresponding $\xi_{\text {STM }}$ value represents the average of 250 scans of the same image. For $\bar{h}>160$ nm (figs. $2 \mathrm{a}-2 \mathrm{c}$ ) two linear portions with a crossing point at $\log L_{\mathrm{s}} \simeq$
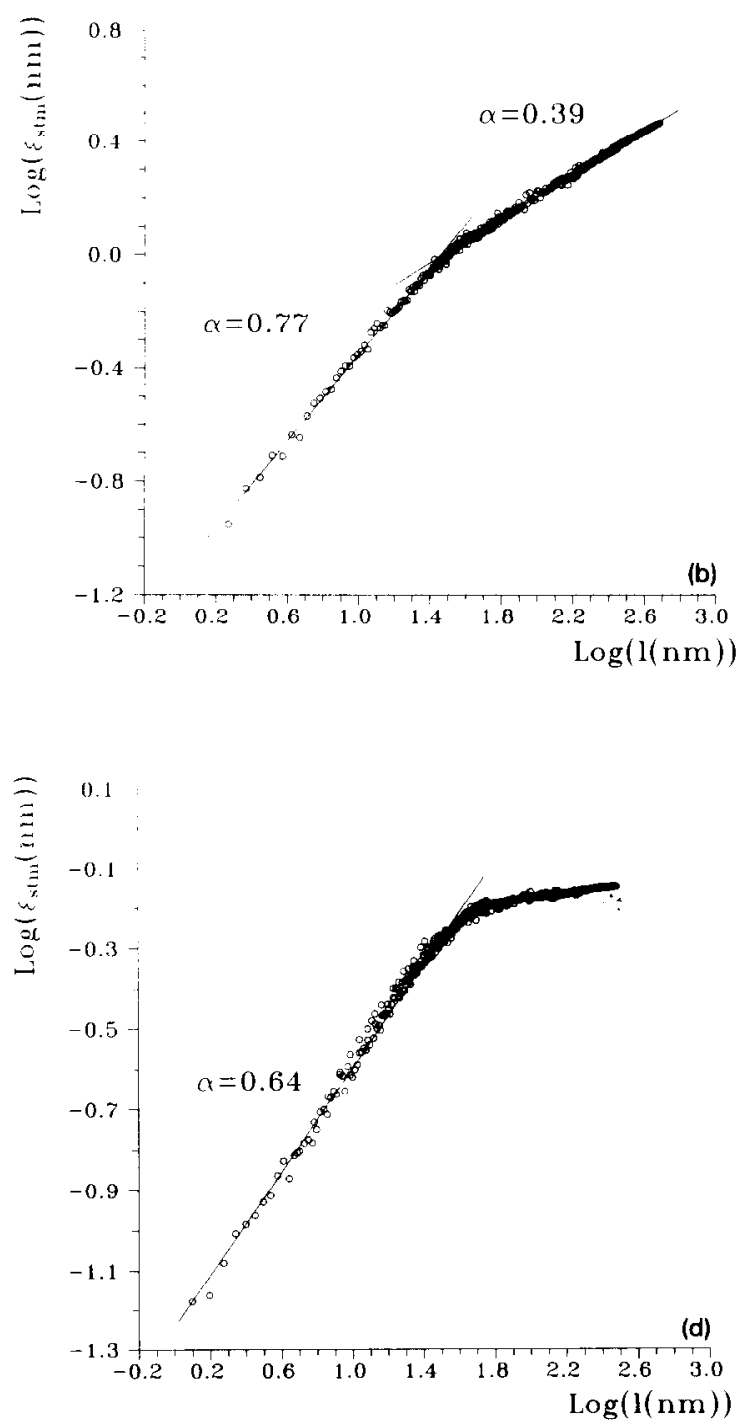

Fig. 2. $\log \xi_{\mathrm{STM}}$ versus $\log L_{\mathrm{s}}$ plots from STM scans obtained for vapour gold films with different values of $\bar{h}$ : (a) $\bar{h}=850 \mathrm{~nm}$; (b) $\bar{h}=500 \mathrm{~nm} ;$ (c) $\bar{h}=170 \mathrm{~nm} ;$ (d) $\bar{h}=30 \mathrm{~nm}$. 
1.5 can be observed. Besides, the slope of the straight lines are $0.71 \pm 0.03<\alpha_{\mathrm{m}}<0.77 \pm 0.03$ for $\log L_{\mathrm{s}}<1.5$, and $0.32 \pm 0.03<\alpha_{\mathrm{m}}<0.39 \pm$ 0.01 for $\log L_{\mathrm{s}}>1.5$. At the highest $L_{\mathrm{s}}$ values a saturation region is approached for $\bar{h}=170 \mathrm{~nm}$ and $\bar{h}=850 \mathrm{~nm}$. It should be noted that for $\bar{h}=170 \mathrm{~nm}$ the $\alpha=0.32$ region extends for a half of a decade, whereas for larger $\bar{h}$ values this zone has one decade of range. This could be explained by the fact that at $\bar{h}=170 \mathrm{~nm}$ the film has not totally reached the steady state $[10,12]$. Accordingly, the average $\alpha$ values should be obtained from the images obtained for samples with $\bar{h}=$ 500 and $850 \mathrm{~nm}$. Thus, from the analysis of 26 images, the average $\alpha_{\mathrm{m}}$ values, $\bar{\alpha}_{\mathrm{m}}$, obtained for $\log L_{\mathrm{s}}<1.5$ and $\log L_{\mathrm{s}}>1.5$ are $\bar{\alpha}_{\mathrm{m}}=0.72 \pm$ 0.07 and $\bar{\alpha}_{\mathrm{m}}=0.38 \pm 0.05$, respectively. It should be noted that $\log L_{\mathrm{s}}=1.5$ corresponds to $L_{\mathrm{s}}=32$ $\mathrm{nm}$ a figure which is close to $d_{\mathrm{s}}$, the average columnar diameter of the deposit surfaces (see (figs. $2 \mathrm{a}^{\prime}-2 \mathrm{~d}^{\prime}$ ).

As shown in fig. 1, for $\bar{\alpha}_{\mathrm{m}}=0.72, \alpha_{\mathrm{mc}}=0.9$ results. Thus, from eq. (1) $D_{\mathrm{s}}$ is close to 2 , i.e., the gold deposit surface behaves as a smooth surface. In fact, there is a trend of columnar surfaces to become smooth as the surface diffusion length of gold atoms at room temperature is sufficiently high to eliminate irregularities of the order of $d_{\mathrm{s}}[10]$. On the other hand, for $L>d_{\mathrm{s}}$ the gold deposit behaves as a rough self-affine
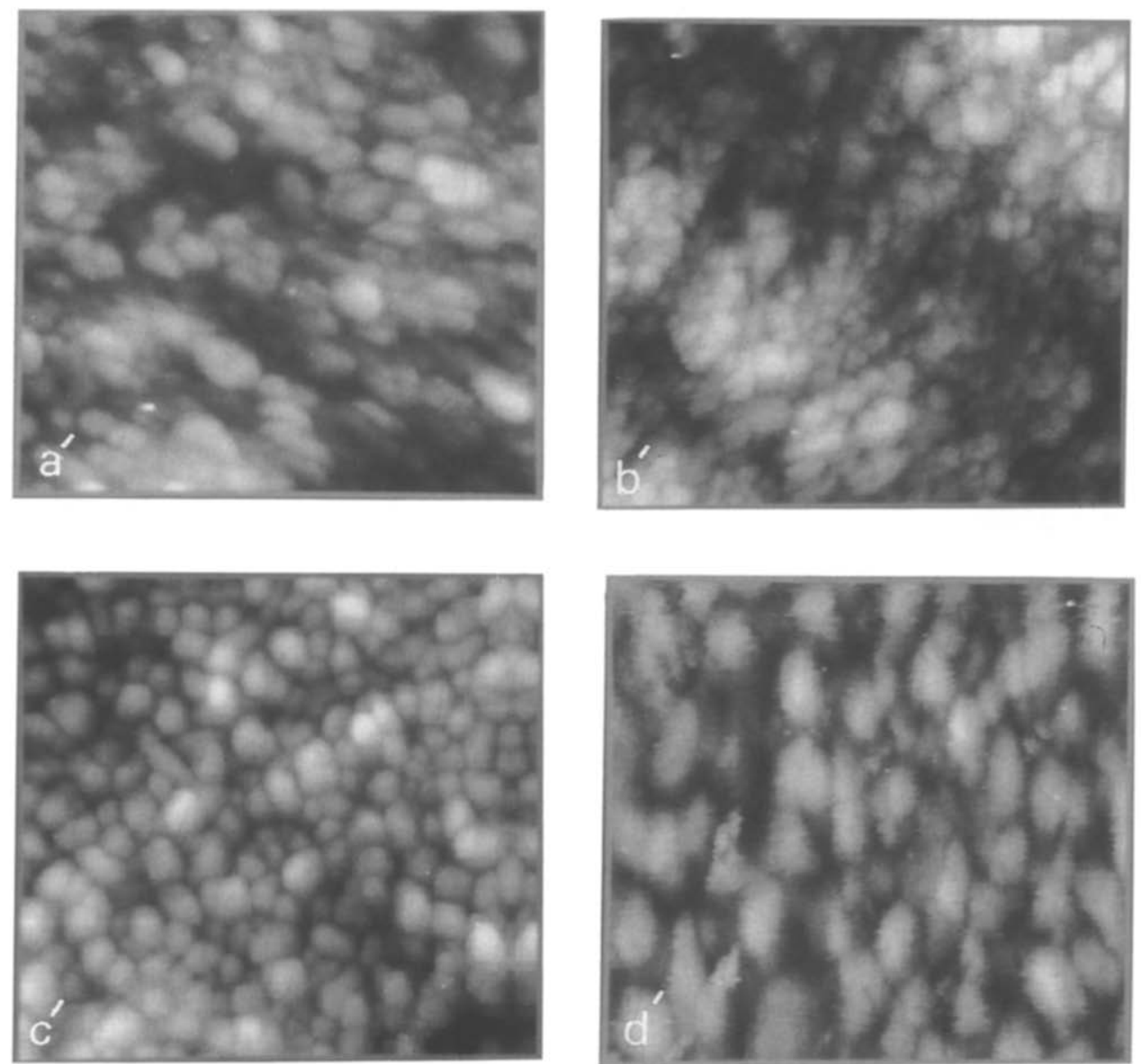

Fig. 2'. Gray-scale STM images (corresponding to figs. $2 \mathrm{a}-2 \mathrm{~d}):\left(\mathrm{a}^{\prime}\right) 507 \times 507 \mathrm{~nm}^{2} ;\left(\mathrm{b}^{\prime}\right) 495 \times 495 \mathrm{~nm}^{2} ;\left(\mathrm{c}^{\prime}\right) 430 \times 430 \mathrm{~nm}{ }^{2} ;\left(\mathrm{d}^{\prime}\right)$ $312 \times 312 \mathrm{~nm}^{2}$. 
fractal surface with $\alpha \simeq 0.38$ and $D_{\mathrm{s}} \approx 2.62$. In this case, as seen in fig. 1 , no correction of $\alpha_{m}$ is required.

The results reported in this work agree with those obtained by using the perimeter $(P)$ versus area $(A)$ method applied to STM images [9]. In this case, two linear portions with a crossing point, $A_{s}$, close to the average columnar size were also observed, and for $A<A_{\mathrm{s}}, \alpha=0.7$; whereas for $A>A_{\mathrm{s}}, \alpha=1 / 3$. Therefore, the values of $\alpha$ obtained by applying either the perimeter versus area method or the dynamic-scaling approach to STM data agree with those obtained from large-scale computer simulations of $3 \mathrm{D}$ deposits generated by the Eden [4] or the ballistic models at incident angles near normal to the substrate without surface restructuring ( $\alpha=1 / 3)$ $[4,12]$.

Finally, for $\bar{h}=30 \mathrm{~nm}$, the $\log \xi_{\text {STM }}$ versus $\log L_{s}$ plot (fig. 2d) shows only a straight line portion with slope $\alpha_{\mathrm{m}}=0.65 \pm 0.02$ extending up to $\log L_{\mathrm{s}}=1.6$, and the trend to reach a saturation region for $\log L_{\mathrm{s}}>1.7 \mathrm{~nm}$. It should be noted that the behaviour of the straight line portion in fig. $2 \mathrm{~d}\left(\alpha_{\mathrm{mc}}=0.78\right)$ is similar to that depicted in figs. $2 \mathrm{a}-2 \mathrm{c}$ for $\bar{h}>160 \mathrm{~nm}$ and $L_{\mathrm{s}}<$ $d_{\mathrm{s}}$. After analyzing 18 images we obtain $\bar{\alpha}_{\mathrm{m}}=$ $0.65 \pm 0.06$ (which leads to $\alpha_{\mathrm{mc}}=0.78$ ). Whereas for the films with $\bar{h}>160 \mathrm{~nm}$ there are significant fluctuations in the $z$-direction, this is not the case for the $30 \mathrm{~nm}$ thick film. The latter can be described as a $2 \mathrm{D}$ film involving the coalescence of smooth hemispherical grains, with $d_{\mathrm{s}} \simeq \bar{h}$, constituting branched gold clusters. Thus, for this case only, the dynamic-scale approach seems not suitable to be applied. However, the independent perimeter versus area analysis can be applied to STM images of this film, as it analyzes it two-dimensionally, yielding $\alpha=0.25$ [9]. This figure agrees with data reported for a $2 \mathrm{D}$ vapour-deposited gold film on $\mathrm{Si}_{3} \mathrm{~N}_{4}$ [14]. The fractal dimension, $D=1.8$ derived from these measurements has been interpreted in terms of percolation gold clusters.
In conclusion, the fractal character of metal surfaces can be determined at the nanometer level from STM data by using either the dynamic-scale approach for surface growth or the perimeter versus area analysis. These procedures appear as powerful tools for testing the validity of different theoretical models on the morphology of thin films and their evolution.

\section{Acknowledgements}

This work was partially supported by CICYT (Spain) under project No. MAT89-0204, R.C.S. and A.J.A. thank financial support from $\mathrm{CON}$ ICET (Argentina).

\section{References}

[1] P. Meakin, Crit. Rev. Solid State Mater. Sci. 13 (1987) 147.

[2] B. Movchan and A.V. Demchishin, Phys. Met. Metallogr. 28 (1969) 83.

[3] R. Messier and J.E. Yehoda, J. Appl. Phys. 58 (1986) 3739.

[4] T. Vicsek, Fractal Growth Phenomena (World Scientific, Singapore, 1989).

[5] P. Pfeifer, Y.J. Yu, M.W. Cole and J. Krim, Phys. Rev. Lett. 62 (1989) 1997.

[6] M.W. Mitchell and D.A. Bonnel, J. Mater. Res. 5 (1990) 2244.

[7] E.A. Eklund, R. Bruinsma and J. Rudnik, Phys. Rev. Lett. 67 (1991) 1759.

[8] R.C. Salvarezza, L. Vázquez, P. Herrasti, P. Ocón, J.M. Vara and A.J. Arvia, Europhys. Lett. 20 (1992) 727.

[9] P. Herrasti, P. Ocón, L. Vázquez, R.C. Salvarezza, J.M. Vara and A.J. Arvia, Electrochim. Acta 37 (1992) 2209.

[10] P. Herrasti, P. Ocón, L. Vázquez, R.C. Salvarezza, J.M. Vara and A.J. Arvia, Phys. Rev. A 45 (1992) 7440.

[11] J.M. Gómez-Rodríguez, L. Vázquez, A.M. Baró, R.C. Salvarezza, J.M. Vara and A.J. Arvia, J. Phys. Chem. 96 (1992) 347.

[12] F. Family, Physica A 168 (1990) 561.

[13] R.F. Voss, in: Fundamental Algorithms in Computer Graphics, Ed. R.A. Earnshaw (Springer, Berlin, 1985).

[14] R.F. Voss, R.B. Laibowitz and E.I. Alessandrini, Phys. Rev. Lett. 49 (1982) 1441. 